\title{
Self-(in)compatibility in 'AS-1', a Local Spanish Almond Cultivar
}

\author{
O. Kodad ${ }^{1}$, A.J Felipe ${ }^{1}$, A. Sánchez ${ }^{2}$, M.M. Oliveira ${ }^{2,3}$ and R. Socias i Company ${ }^{1}$ \\ ${ }^{1}$ Unidad de Fruticultura, CITA de Aragón, Av. Montañana 930, 50059 Zaragoza, Spain \\ ${ }^{2}$ Instituto de Tecnologia Química e Biológica (ITQB), Instituto de Biologia Experimental \\ e Tecnológica (IBET), Quinta do Marquês, 2784-505 Oeiras. Portugal \\ ${ }^{3}$ Universidade de Lisboa, Faculdade de Ciencias, Dep. Biologia Vegetal, Campo Grande, \\ 1749-016 Lisbon, Portugal \\ rsocias@aragon.es
}

Keywords: Prunus amygdalus, PCR, pollen tube growth, fruit set

\begin{abstract}
The finding of new self-compatible cultivars in local population is a breeder's objective in order to increase the sources of self-compatibility for the almond (Prunus amygdalus Batsch) breeding programmes. 'AS-1', a local Spanish selection, was considered to be self-compatible according to its self pollen tube growth and was incorporated in the almond breeding programme of Zaragoza as a source for this trait. However, both pollen tube growth and fruit set after self-pollination have shown that this selection is self-incompatible. The PCR analysis using specific and consensus primers revealed that its genotype is $S_{8} S_{12}$, where both alleles control selfincompatibility and do not confer self-compatibility in almond. The field crosses of 'AS-1' and 'Marcona Flota', of the same $S$ genotype, were incompatible, confirming the $S$ genotype of 'AS-1' determined by PCR analysis. The sequencing of the SFB $S$ haplotype showed that 'AS-1' presents $\mathrm{SFB}^{8}$ and $\mathrm{SFB}^{12}$. Moreover, the absence of any notable deletion or insertion upstream from the HVa and HVb regions in the sequence of the $\mathrm{SFB}^{8}$ and $\mathrm{SFB}^{12} S$-haplotypes confirm their identity. All these results, including pollination tests, PCR analysis and cloning and sequencing of the $S$ alleles of 'AS-1', indicate that this local selection is self-incompatible.
\end{abstract}

\section{INTRODUCTION}

The limited number of sources for self-compatibility utilised in almond breeding programmes (Socias i Company, 2002) recommended the search for new self-compatible forms among the different almond populations, mainly in local forms, such it happened with the local cultivars of the Puglia region in Italy (Grasselly and Olivier, 1976). As a result of an initial search, 'AS-1' was noticed in Tamarite de la Litera, Huesca province, Spain, as a single late-blooming tree, isolated from other simultaneously blooming almond trees and setting fruit every year, thus presumably self-compatible. Herrero and Felipe (1975) conducted controlled field pollinations and pollen tube growth observations in several cultivars and reported that 'AS-1' was self-compatible.

'AS-1' was included in the Zaragoza breeding programme and Socias i Company and Felipe (1988) reported a 3:1 (SC:SI) distribution in the progeny of the cross 'Tuono' $\times$ 'AS1', as expected in a cross involving two different heterozygous self-compatible cultivars. When 'AS-1' was open-pollinated, these authors found four self-compatible seedlings in a progeny of 15 , thus presumably showing a self-compatible behaviour both in expression and transmission. 
Thus the aim of this work was to confirm the molecular identity of the $S$-RNases and SFBs of 'AS-1', as well as the phenotypic expression of its self-(in)compatibility by pollen tube growth studies after controlled pollinations.

\section{MATERIALS AND METHODS Plant Material}

Two Spanish cultivars were studied, 'AS-1' and 'Marcona'. Plant samples were obtained from the Spanish almond germplasm collection located at the CITA de Aragón in Zaragoza, maintained as living plants grafted on the almond $\times$ peach hybrid clonal rootstock INRA GF-677 using the standard management practices (Espiau et al., 2002). Pollen of 'Marcona Flota' was obtained from CEBAS (CSIC), Murcia (Spain).

\section{Cloning and g DNA Sequencing of S-RNase and SFB S-haplotype}

Genomic DNA was extracted from leaves following the method based on Doyle and Doyle (1987). For PCR amplification the forward primer PaConsI-F and the reverse primer EM_PCconsRD were used according to Ortega et al. (2006). A fragment of the SFB was PCR-amplified according to Yamane et al. (2003) with primers SFB-C1F and SFB-FB3 (Ushijima et al., 2003). Prior to cloning, the band sizes corresponding to the target alleles were purified and quantified on $1.5 \%$ agarose gel. The purified PCR products were cloned into the vector pCR2.1 using the TA Cloning Kit.

\section{Pollination Tests}

Physiological self-compatibility was assessed by pollen tube growth and fruit set after artificial hand pollination. Self- and cross-compatibility was assessed in the laboratory by observation of pollen tube growth in the style under a fluorescence microscope. Fruit set was evaluated in the field on several branches, where at least 100 flowers per branch were emasculated and self-pollinated. In other branches, the flowers were cross-pollinated with 'Marcona' and 'Marcona Flota' pollen. Fruit set was recorded in June for all treatments.

\section{RESULTS AND DISCUSSION}

PCR amplification of 'AS-1' genomic DNA using primers PaConsI-F and EM_PCconsRD produced only one fragment (Fig 1). The cloning and sequencing analysis revealed that this band corresponds to the $S_{12}$ allele, confirming the results previously obtained by Kodad et al. (2008). For the other allele, we assume that it is identical to $S_{8}$ sequenced in 'Nonpareil' by Ushijima et al. (1998), since the specific primer designed for the $S_{8}$ allele from the second intron of 'Nonpareil' amplifies a similar band of 640 bp in 'AS-1' (Fig 2).

Self-compatibility observed in some genotypes of several Prunus species has been attributed to a defective function of the pollen, which could be due to a mutation, deletion or insertion in the coding region of the SFB $S$-haplotype, such as in $P$. avium and $P$. mume (Ushijima et al., 2004). The sequence analysis of the amplified fragment from 'AS$1^{\prime}$ showed that it is identical to the $\mathrm{SFB}^{8}$ and $\mathrm{SFB}^{12} S$-haplotypes, excluding the presence of the $\mathrm{SFB}^{\mathrm{f}} S$-haplotype. Moreover, the results obtained in the present work indicate the absence of any notable deletion or insertion upstream from the $\mathrm{HVa}$ and $\mathrm{HVb}$ regions in the sequence of the $\mathrm{SFB}^{8}$ and $\mathrm{SFB}^{12} S$-haplotypes.

No pollen tubes were observed at the style base after self-pollination in the laboratory and no fruit set was obtained after self-pollination in the field, indicating that 
'AS-1' is self-incompatible. The high percentage of pistils with pollen tubes at the style base after pollination with 'Marcona' pollen shows that 'AS-1' pistils are able to sustain pollen tube growth if this pollen is cross-compatible. Cross-incompatibility of 'AS-1' and 'Marcona Flota', with the same $S$ genotype $\left(S_{8} S_{12}\right)$, agrees with the molecular data.

Herrero and Felipe (1975) incorrectly reported that 'AS-1' was self-compatible after controlled pollinations and pollen tube growth tests, with more than $74 \%$ of flowers with pollen tubes in the ovary, in disagreement with our results, but they did not obtain any fruit set when 'AS-1' was selfed, corroborating our results.

As a conclusion, based on the study of pollen tube growth, fruit set after selfpollination, PCR analysis and sequencing of the $S$-RNases and the SFB $S$-haplotypes, 'AS-1' must be reclassified as self-incompatible. The cross-incompatibility between 'AS1 ' and 'Marcona Flota' corroborates the new Cross Incompatible Group XXI proposed by Kodad et al. (2007).

\section{ACKNOWLEDGMENTS}

This work was supported by project AGL2007-65853-C02-02 of the Spanish CICYT. O. Kodad gratefully acknowledges a scholarship from the Spanish INIA and of the Consolidated Research Group A12 of Aragón.

\section{Literature Cited}

Doyle, J.J. and Doyle, J.L. 1987. A rapid DNA isolation procedure for small quantities of fresh tissue. Phytochem. Bull. 19:11-15.

Espiau, M.T., Ansón, J.M. and Socias i Company, R. 2002. The almond germplasm bank of Zaragoza. Acta Hort. 591:275-278.

Herrero, M. and Felipe, A.J. 1975. Pollinisation de l'amandier. Incompatibilité pollenstyle. $2^{\mathrm{e}}$ Colloque GREMPA, Montpellier-Nîmes, Septembre 1975.

Grasselly, C. and Olivier, G. 1976. Mise en évidence de quelques types autocompatibles parmi les cultivars d'amandier ( $P$. amygdalus Batsch) de la population des Pouilles. Ann. Amélior. Plant. 26:107-113.

Kodad, O. and Socias i Company, R. 2007. Review and update of self-incompatibility alleles in almond. Acta Hort. 814: 421-424.

Kodad, O., Alonso, J.M., Sánchez, A., Oliveira, M.M. and Socias i Company, R. 2008. Evaluation of genetic diversity of $S$-alleles in an almond germplasm collection. J. Hort. Sci. Biotechnol. 83:603-608.

Ortega, E., Bošković, R., Sargent, D.J. and Tobutt, K.R. 2006. Analysis of S-RNase alleles of almond (Prunus dulcis): characterization of new sequences, resolution of synonyms and evidence of intragenic recommendation. Mol. Genet. Genomics 276:413-426.

Socias i Company, R. 2002. Latest advances in almond self-compatibility. Acta Hort. 591:205-211.

Ujishima, K., Sassa, H., Dandekar, A.M., Gradziel, T.M., Tao, R. and Hirano, H. 2003. Structural and transcriptional analysis of the self-incompatibility locus of almond: identification of a pollen-expressed F-Box gene with haplotype-specific polymorphism. Plant Cell 15:771-781.

Ushijima, K., Yamane, Y., Watari, A., Kakehi, E., Ikeda, K., Huak, R.H., Iezzoni, A.F. and Tao, R. 2004. The S-haplotype-specific F-Box protein gene, SFB, is defective in self-compatible haplotypes of Prunus avium and P. mume. Plant J. 39:573-586. 


\section{$\underline{\text { Figures }}$}

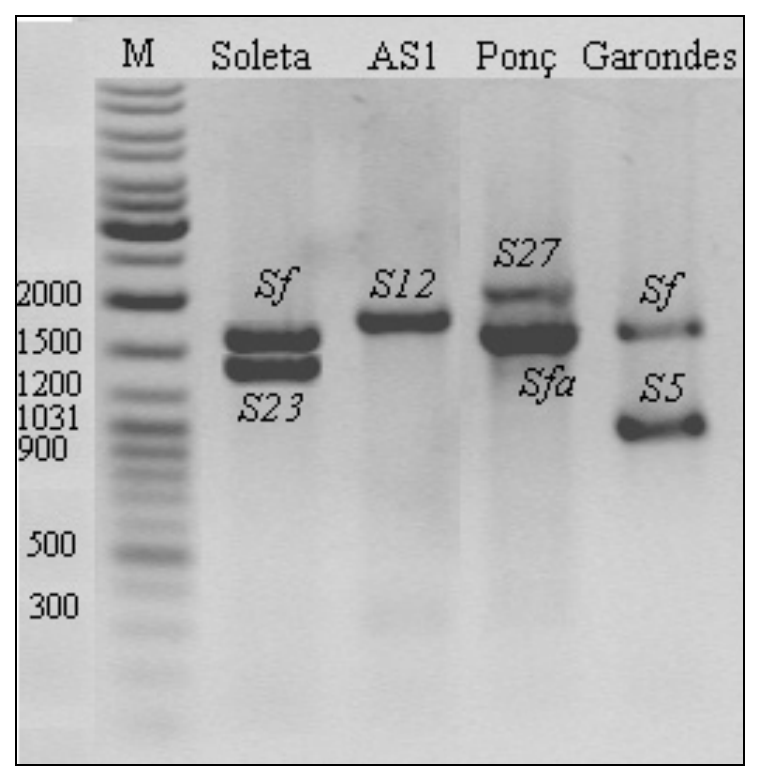

Fig. 1. S-genotype identification in several almond cultivars amplified with the primer pair PaConsI-F and EM_PCconsRD. M: 1kb Ladder (Fermentas).

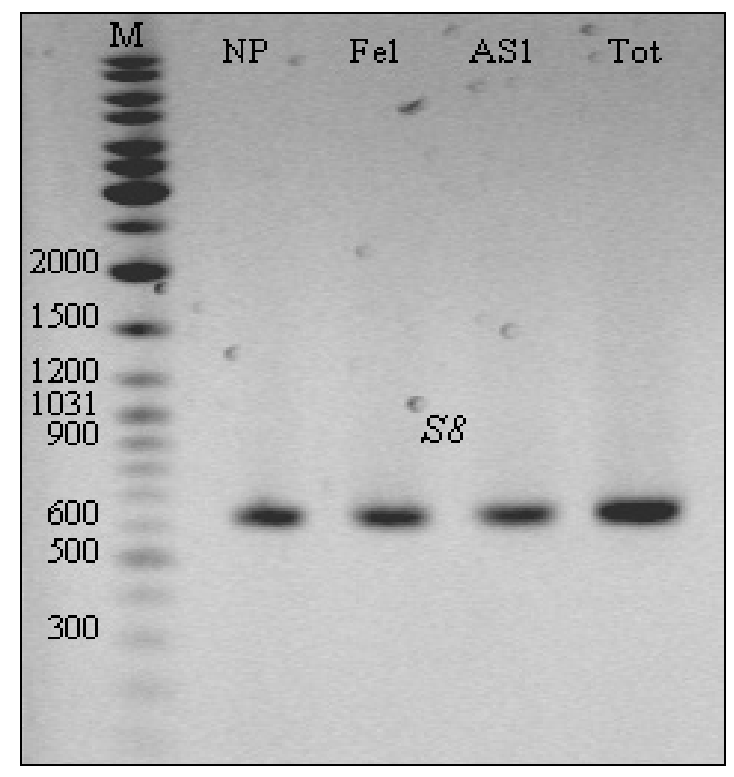

Fig. 2. Agarose (1.5\%) gel showing $S$-allele fragments amplified with the specific $S_{8}$ primers in several almond cultivars. NP: Nonpareil $\left(S_{7} S_{8}\right)$. Fel: Felisia $\left(S_{f} S_{8}\right)$. AS-1 $\left(S_{8} S_{12}\right)$. Tot: Totsol $\left(S_{8} S_{31}\right)$. M: $1 \mathrm{~kb}$ Ladder (Fermentas) 